\title{
Silencing GhAGPL1 Reduces the Quality and Quantity of Corms and Cormels in Gladiolus
}

\author{
Shanshan Seng ${ }^{1}$, Jian Wu $\mathbf{1}^{1}$, Jiahui Liang, Fengqin Zhang, Qiuyan Yang, Junna $\mathrm{He}^{2}$, and \\ Mingfang $\mathbf{Y i}^{2}$ \\ Beijing Key Laboratory of Development and Quality Control of Ornamental Crops, Department of \\ Ornamental Horticulture and Landscape Architecture, China Agricultural University, \\ Yuan Mingyuan Western Road No. 2, Beijing 100193, China
}

\begin{abstract}
AdDitional INDEX words. gene silencing, starch biosynthesis, cut flower, large subunit, Gladiolus hybridus
Abstract. Starch accumulation is important during com development. ADP-glucose pyrophosphorylase (AGPase) is the rate-limiting enzyme in starch synthesis. AGPL is the large subunit of AGPase. Here, we isolated and characterized the large subunit of AGPase gene GhAGPL1 in gladiolus (Gladiolus hybridus). GhAGPL1 was highly expressed in sink organs (cormels and corms). The expression of GhAGPL1 was induced by glucose, sucrose, and mannitol, and it was repressed by abscisic acid (ABA). Overexpression of GhAGPL1 in the arabidopsis (Arabidopsis thaliana) apl1 mutant resulted in complementation of AGPase activity and thus starch synthesis. Silencing GhAGPL1 in gladiolus decreased the transcript level of GhAGPL1 and GhSus, and resulted in the reduction of AGPase activity and starch content in gladiolus corm and cormel. Meanwhile, sucrose content was higher in GhAGPL1-silenced corm. Surprisingly, silencing GhAGPL1 in gladiolus produced smaller corms and fewer number of cormels. Overall, GhAGPL1 contributed to the quality and quantity of gladiolus corms and cormels.
\end{abstract}

Worldwide, gladiolus is a commercially sold cut flower, whose sales rank the second highest among bulb flowers ( $\mathrm{Wu}$ et al., 2015). Corms and cormels are the only materials used for commercial propagation. Yearly, gladiolus produces new corms and cormels, whereas the mother corm undergoes atrophy. The new corm forms at the shoot base, just above the old corm (mother corm); then, after 11 weeks, stolons develop from the axillary buds situated between the mother and new corms; finally, the cormels are formed at the tips of branched stolons in weeks 14 to 17 (Chen and Yi, 2004). The new corm (diameter $>2.4 \mathrm{~cm}$ ) is used for the production of cut flowers. The quality of corms is correlated with flowering and corm yield (Sharma and Gupta, 2003). The cormel is used for new corm reproduction. Therefore, the quality of the cut flower is determined by the quality of the corm, and the production of cut flower is determined by the quantity of corms and cormels. However, corm degeneration is a major problem in gladiolus commercial cultivation. Thus, the production of cut flowers benefits from improving the yield of corms and cormels.

Studies have shown that phytohormones like ABA, gibberellin, cytokinins, and auxins are involved in corm development (Dantu and Bhojwani, 1995). Macronutrients, such as nitrogen and phosphorus, also contribute to corm and cormel yields (Butt, 2005). In addition, starch accounts for half of dry weight in corms (Seng et al., 2016). Starch is a major reserve carbohydrate, and also functions to balance source and sink metabolites ( $\mathrm{Yu}$ et al., 2015). Sucrose produced in leaves is transported into underground organs (new corm and cormels) where it is used

Received for publication 13 Oct. 2016. Accepted for publication 10 Feb. 2017. We thank Doctoral Fund of Ministry of Education of China (20120008110006) and National Natural Science Foundation Projects (31171991) for their support. We also thank Carina Carianopol $\mathrm{PhD}$ candidate in Biology Science at University of Toronto; E-mail: carina.carianopol@mail.utoronto.ca) for help in revising the manuscript.

${ }^{1}$ These authors contributed equally to this work.

2Corresponding authors. E-mail: hejunna@cau.edu.cn or ymfang@cau.edu.cn. for starch synthesis by AGPase. Starch synthesis and accumulation affect the growth of underground organs, including corm enlargement, stolon swelling, and the production of cormels.

AGPase is a rate-limiting enzyme in starch synthesis, which catalyzes the conversion of glucose-1-phosphate (Glc1-P) and ATP to pyrophosphate and ADP-glucose (Kang et al., 2013; Seng et al., 2016). In higher plants, AGPase functions as a heterotetramer, with two different subunits (large subunits AGPL and small subunits AGPS). AGPS is proposed to play a dominant role in catalysis. Many studies have shown that both AGPL and AGPS influence catalytic and allosteric regulatory properties of the enzyme, suggesting diverse catalytic properties exist in higher plants. AGPLs are involved in seed development (Tuncel et al., 2014a), thermotolerance (Saripalli and Gupta, 2015), redox (Tuncel et al., 2014b), and tuberization (Müller-Röber et al., 1992). However, $A G P L$ function in corm development is not yet well characterized.

The large subunits of AGPase, AGPLs, are proposed to have evolved by divergence, specialization, and subfunctionalization from a common ancestor (Ventriglia et al., 2008). Different large subunits confer distinct regulatory properties to AGPase, according to the necessities for starch synthesis in a given tissue (Crevillén et al., 2003). In arabidopsis, AGPL1 is the main large subunit in source tissues, whereas AGPL2 and AGPL3 are the main isoforms in sink tissues (Crevillén et al., 2005). In addition, different large subunit shows different response to sugar, light, and osmotic agents (Crevillén et al., 2005; Sokolov et al., 1998).

In this study, we isolated GhAGPL1 gene from gladiolus corm. We explored whether GhAGPL1 can function in arabidopsis, and how it influences gladiolus corm development. Our results provide definitive evidence that starch biosynthesis during corm development is regulated by GhAGPL1. Moreover, silencing GhAGPL1 reduced the quality and quantity of gladiolus corms and cormels. 


\section{Materials and Methods}

Plant materials and growth Conditions. Gladiolus (cv. Rose Supreme) was planted in the Science Research Garden at China Agricultural University. Organs were taken from 25-weekold plants to detect GhAGPL1 expression. Cormels $(0.6-1.0 \mathrm{~cm}$ diameter) were cultured at $22{ }^{\circ} \mathrm{C}$ in growth chamber with $16 / 8 \mathrm{~h}$ light/dark photoperiod, with light intensity of $54 \mu \mathrm{mol} \cdot \mathrm{m}^{-2} \cdot \mathrm{s}^{-1}$. For abiotic stress, cormels were placed in a petri plate in sugarfree medium containing $200 \mathrm{~mm}$ mannitol, $200 \mathrm{~mm}$ glucose, $200 \mathrm{~mm}$ sucrose, or $100 \mu \mathrm{M} \mathrm{ABA}$ in the dark for $36 \mathrm{~h}$.

Arabidopsis seeds were sterilized with $1 \%(\mathrm{v} / \mathrm{v}) \mathrm{NaClO}$ for $20 \mathrm{~min}$, washed five times with sterile water, and placed on Murashige and Skoog (MS) medium. Following stratification for $3 \mathrm{~d}$ at $4{ }^{\circ} \mathrm{C}$ in the dark, the seeds were transferred into a growth chamber and were germinated at $22{ }^{\circ} \mathrm{C}$ with $16 / 8 \mathrm{~h}$ light/ dark photoperiod, under $50 \mu \mathrm{mol} \cdot \mathrm{m}^{-2} \cdot \mathrm{s}^{-1}$ light intensity. Seedlings were transferred to forest soil and vermiculite $(1: 1 \mathrm{v} / \mathrm{v})$ in the growth chamber after $7 \mathrm{~d}$. Nicotiana benthamiana seeds were planted into pots and cultured under the same conditions as the arabidopsis.

ISOLATION AND SEQUENCE ANALYSIS OF GHAGPL1. RNA extraction kit (Tiangen, Beijing, China) was used to extract total RNA from gladiolus cormels. Subsequently, cDNA was synthesized by M-MLV reverse transcriptase (TaKaRa, Dalian, China) with a $3^{\prime}$ adaptor [5'-CCAGTGAGCAGAGTGAC-

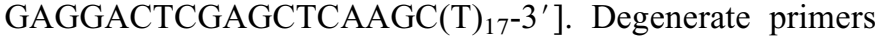
were designed for GhAGPL1 cloning based on conserved regions of homologous $A G P L 1$ from other species. The amplified product was used to clone the full-length GhAGPL1 by the rapid amplification of cDNA ends (RACE) according to the manufacturer's protocol (Clontech, Mountain View, CA). All primers are listed in Supplemental Table 1.

ClustalX1.8 (Thompson et al., 1997) and BioEdit7.0 (Hall, 1999) were used to perform multiple amino acid alignments, and phylogenetic tree was constructed by the neighbor-joining method using the MEGA5.0 software (Tamura et al., 2011). Accession numbers for public sequences used in the phylogenetic analysis are listed in Supplemental Table 2.

Subcellular location of GhaGPL1-GFP Fusion PRoteins. The full length of GhAGPL1 coding region was digested by SalI/KpnI and subcloned into pCAMBIA1300-GFP vector to generate pCAMBIA1300-GhAGPL1-GFP expression plasmid. The GhAGPL1-GFP plasmid was transformed into onion (Allium cepa) epidermal cells by previously described particle bombardment method (Wang and Fang, 2002). The GhAGPL1-GFP fusion protein transformed into onion cells was microscopically detected with a light microscope (Eclipse TE2000-Econfocal; Nikon, Melville, NY). Then, images were analyzed using EZ-C1 software (Nikon).

EXPRESSION ANALYSIS OF GHAGPL1 BY QUANTITATIVE REALTIME POLYMERASE CHAIN REACTION. Primers for quantitative realtime polymerase chain reaction (qRT-PCR) system (StepOne; Applied Biosystems, Foster City, CA) are listed in Supplemental Table 1. GhActin and AtActin2 were used as internal control for gladiolus and arabidopsis, respectively. Relative expression was calculated by the $2^{-\triangle \Delta \mathrm{CT}}$. The detailed procedures were described previously (Livak and Schmittgen, 2001). Each qRT-PCR assay was run using three biological replicates.

Determining AGPase activity, starch, and sucrose CONTENT. For AGPase activity, samples were flash frozen using liquid nitrogen. About $0.05 \mathrm{~g}$ of frozen powder was resuspended on ice with $500 \mu \mathrm{L}$ extraction buffer [ $1 \mathrm{~mm}$ ethylenediaminetetraacetic acid, $50 \mathrm{~mm}$ 4-(2-hydroxyethyl)-1-piperazineethanesulfonic acid (pH 7.5), $5 \mathrm{~mm}$ dithiothreitol, $5 \mathrm{~mm} \mathrm{MgCl}_{2}, 20 \%$ sucrose, and freshly added $1 \mathrm{~mm}$ phenylmethylsulfonyl fluoride, $1 \mu \mathrm{g} \cdot \mathrm{mL}^{-1}$ pepstatin, and $1 \mu \mathrm{g} \cdot \mathrm{mL}^{-1}$ leupeptin]. The slurry was centrifuged at $13,400 g_{\mathrm{n}}$ for $15 \mathrm{~min}$ at $4{ }^{\circ} \mathrm{C}$, and the supernatant was immediately used for AGPase enzyme assay (Cross et al., 2004). Three biological replicates were used in each experiments.

A previously described method was used for determining sucrose and starch content (Hendriks et al., 2003). Pulverized material $(0.02 \mathrm{~g})$ was extracted with $250 \mu \mathrm{L}$ ethanol $(80 \%$ ethanol twice, followed by $50 \%$ ethanol once). After that, the samples were heated at $90^{\circ} \mathrm{C}$ for $30 \mathrm{~min}$. The supernatants were collected in another tube. The pellets were reextracted twice more, using the same procedure. The combined supernatants were used for the determining sucrose content as described previously (Hendriks et al., 2003). For starch quantification, the pellets from the ethanol extraction were incubated at $95^{\circ} \mathrm{C}$ in $0.1 \mathrm{M} \mathrm{NaOH}$ for $30 \mathrm{~min}$ and neutralized with $\mathrm{HCl} /$ sodium acetate $(0.5 \mathrm{M} \mathrm{HCl}+0.1 \mathrm{M}$ acetate/ $\mathrm{NaOH}, \mathrm{pH} 4.9)$. Suspensions $(50 \mu \mathrm{L})$ were digested overnight with amyloglucosidase and $\alpha$-amylase.

IODINE STAINING OF STARCH. Leaves from arabidopsis or gladiolus were incubated in $75 \%$ ethanol at $80{ }^{\circ} \mathrm{C}$ until chlorophyll was totally eliminated. Then, treated leaves were rinsed with distilled water and were stained with Lugol's solution $\left(0.06 \% \mathrm{I}_{2}, 0.1 \% \mathrm{KI}\right.$, and $\left.4 \mathrm{~mm} \mathrm{HCl}\right)$ for $10 \mathrm{~min}$. Finally, the stained leaves were rinsed with distilled water for $15 \mathrm{~min}$ (Caspar et al., 1985).

Complementation of apl1 mutant with GhaGPL1. Arabidopsis apll mutant plants (Salk_139228) were transformed using the floral dip method (Clough and Bent, 1998) with Agrobacterium tumefaciens GV3101 harboring 35S:GFPGhAGPL1/pCAMBIA1301. Infected seeds were screened on MS medium containing $50 \mu \mathrm{g} \cdot \mathrm{mL}^{-1}$ hygromycin. Lines with homozygous seeds were used for related assays.

Silencing of GhAGPL1 in Gladiolus. Silencing of GhAGPL1 by virus-induced gene silencing (VIGS) was performed as described previously (Zhong et al., 2014). Cormels with 6 to $10 \mathrm{~mm}$ in diameter were used for VIGS assays. First, a 313-base pair (bp) specific fragment of GhAGPL1 were generated and cloned into pTRV2 vector. TRV1, TRV2, and TRV2-GhAGPL1 were transformed individually into agrobacterium GV3101. The transformed agrobacterium colonies were cultured overnight in Luria-Bertani medium supplemented with $50 \mu \mathrm{g} \cdot \mathrm{mL}^{-1}$ kanamycin and $100 \mu \mathrm{g} \cdot \mathrm{mL}^{-1}$ rifampicin. After overnight culture, the agrobacterium lines were collected and resuspended in infiltration buffer $\left[10 \mathrm{~mm} \mathrm{MgCl}_{2}, 200 \mathrm{~mm}\right.$ acetosyringone, and $10 \mathrm{~mm}$ 2-(N-morpholino) ethanesulfonic acid ( $\mathrm{pH} 5.6)]$ to a final $\mathrm{OD}_{600}$ of 1.8. Equal volumes of TRV1 and TRV2 (used as the control), as well as TRV1 and TRV2GhAGPL1 were mixed together and placed at room temperature for $3 \mathrm{~h}$ in the dark before vacuum infiltration. Young plants were submerged in infiltration buffer and were subjected to vacuum infiltration under $0.7 \mathrm{MPa}$ for $20 \mathrm{~min}$. After infiltration, the plants were transferred into pots containing a mixture of $1: 1(\mathrm{v} / \mathrm{v})$ soil:sand mixture and grown in a greenhouse $\left(22{ }^{\circ} \mathrm{C}\right.$, relative humidity $60 \%$ to $70 \%, 16 / 8 \mathrm{~h}$ light/dark cycle).

Transmission ELECTRON MICROSCOPY. The leaves and corms from plants cultured in a $16 / 8 \mathrm{~h}$ light/dark photoperiod were collected at the midpoint of the light period. Small pieces $\left(2 \mathrm{~mm}^{2}\right)$ were immediately fixed by submersion in a cacodylate 
buffer $[0.05$ м sodium cacodylate and $3 \%(\mathrm{v} / \mathrm{v})$ glutaraldehyde, $\mathrm{pH}$ 7.4] for $3 \mathrm{~h}$ at $4{ }^{\circ} \mathrm{C}$, under vacuum. After that, the specimens were washed three times in a solution of $1 \%$ sucrose in $0.05 \mathrm{~m}$ sodium cacodylate buffer, for $30 \mathrm{~min}$ each at $4{ }^{\circ} \mathrm{C}$, and postfixed with a solution of $1 \%$ osmium tetroxide in $0.05 \mathrm{~m}$ sodium cacodylate buffer, then kept overnight for complete removal of fixative at $4{ }^{\circ} \mathrm{C}$. After two washes of 30 min each at $4{ }^{\circ} \mathrm{C}$ with the same cacodylate buffer, the samples were transferred into $30 \%, 50 \%$, and $70 \%$ ethanol for $10 \mathrm{~min}$ each at $4{ }^{\circ} \mathrm{C}$ to dehydrate. Then they were dehydrated with $1 \%$ uranyl acetate in $70 \%$ ethanol for $16 \mathrm{~h}, 90 \%$ ethanol for $10 \mathrm{~min}, 96 \%$ ethanol for $30 \mathrm{~min}$, and $100 \%$ ethanol for $2 \mathrm{~h}$, with one change after the first hour at $4{ }^{\circ} \mathrm{C}$. London Resin White (London Resin, Reading, UK) infiltration and polymerization at $60{ }^{\circ} \mathrm{C}$ were performed as previously described (Gonzalez-Melendi et al., 2008). Ultra-thin (70 to $90 \mathrm{~nm}$ ) sections were stained as described previously by Roldan et al. (2007).

\section{Results}

ISOLATION AND SEQUENCE ANALYSIS OF GHAGPL1. GhAGPL1 was isolated using the RACE method. The length of GhAGPL1 gene was 1919 bp with a 1554-bp open reading frame (ORF). GhAGPL1 encodes a 517 amino acid residues protein, with a calculated molecular mass of $56.5 \mathrm{kDa}$ and an isoelectric point of 7.47.

To further investigate the GhAGPL1 protein, we used MEGA5.0 for phylogenetic analysis. The results (Fig. 1A) suggested that GhAGPL1 is more similar to homologs in monocotyledons such as Zea mays, Oryza sativa, Triticum aestivum, and Hordeum vulgare rather than dicotyledons. Sequence analysis (Fig. 1B) indicated that GhAGPL1 shares four conserved domains [motif $(a)$ to $(d)$ : ATP-binding motif, catalytic motif, Glc-1-P binding motif, and activator site] with other homologs.

SubCEllular localization of GHAGPL1 IN ONION EPIDERMAL CELLS. To examine the subcellular localization of GhAGPL1, the ORF

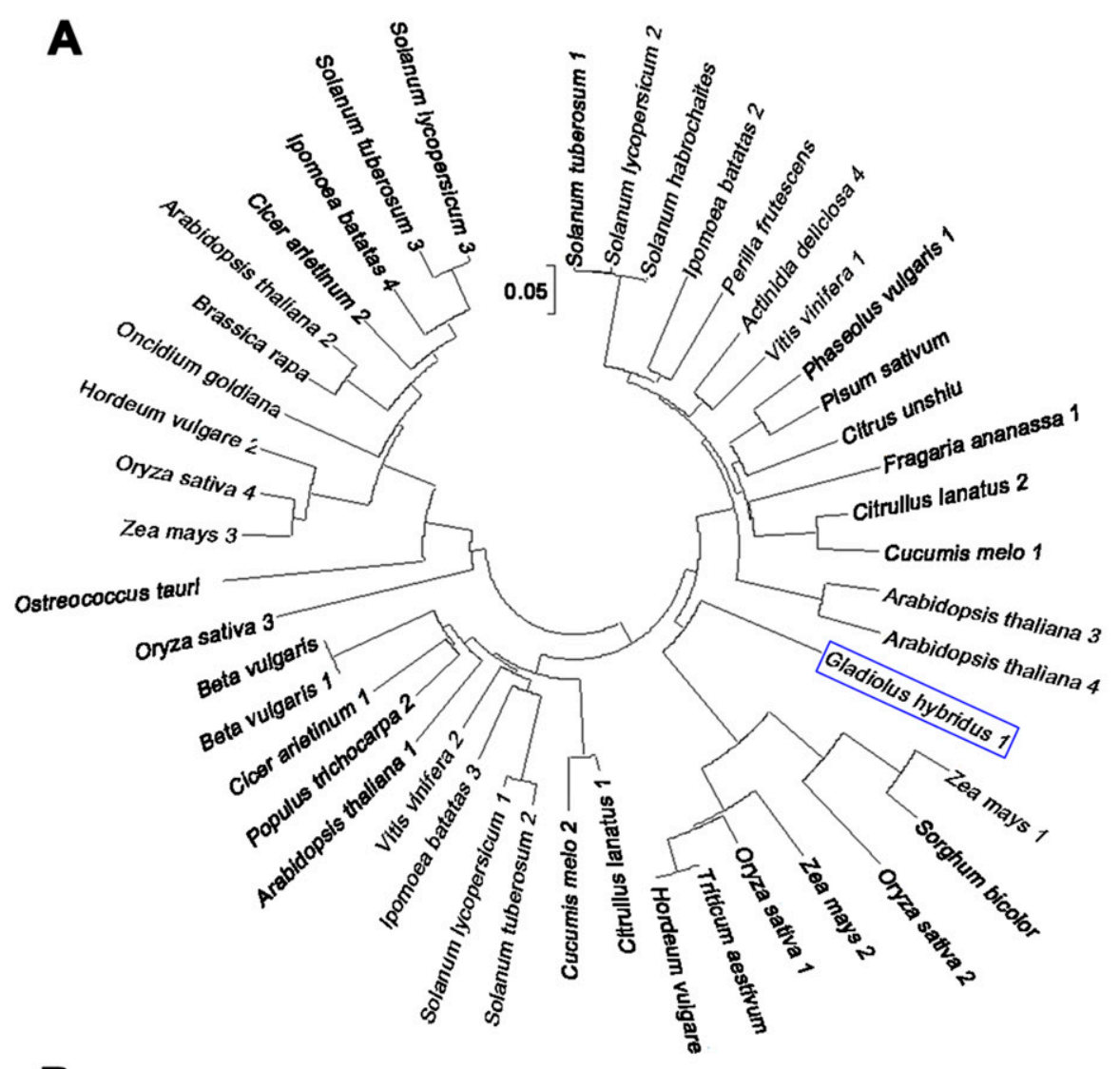

B

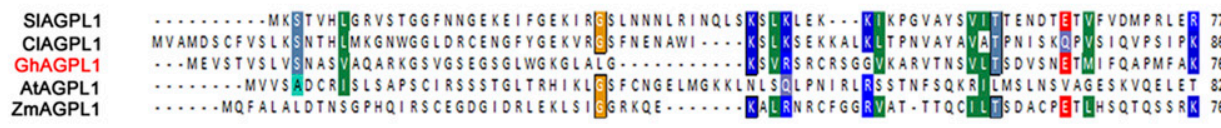

A.
AtAGPL1
ZMAGPL1

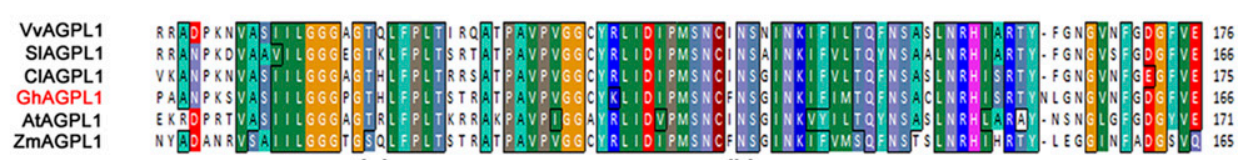

ZmAGPL1

(a)

(b)

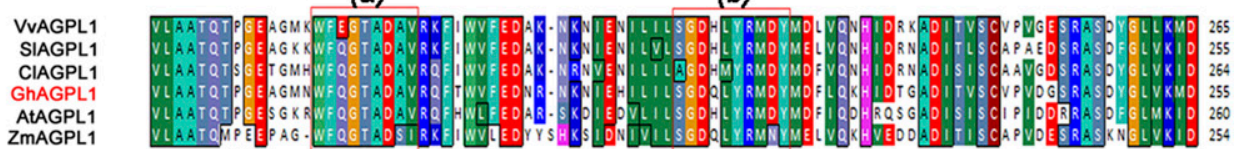

AtAGPL1

ZmAGPL1

(c)

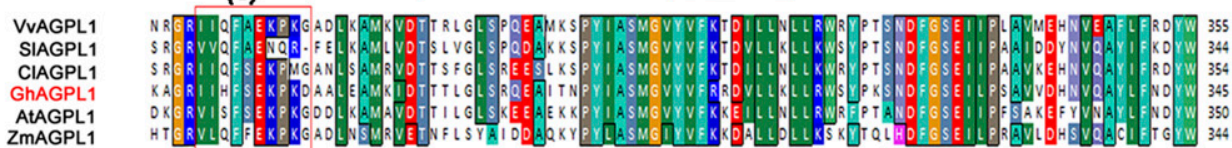

AtAGPL1

ZmAGPL1

VVAGPL1

SIAGPL1

CIAGPL1

AtAGPL1

AtAGPL1
ZmAGPL1

VVAGPL1

SIAGPL1

CIAGPL1

AtAGPL1

AtAGPL1

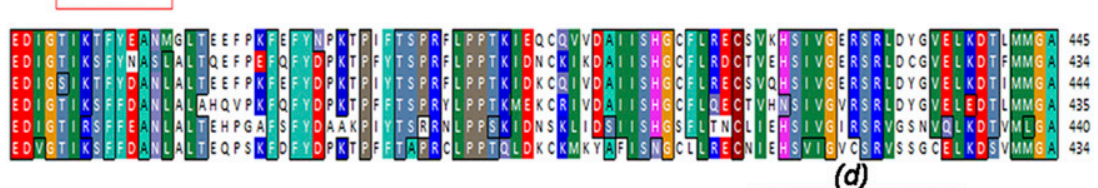

(d)

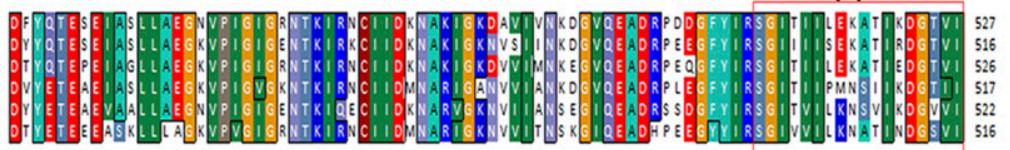

Fig. 1. Phylogenetic analysis and sequence alignment of gladiolus GhAGPL1 protein. (A) Phylogenetic analysis of GhAGPL1 and AGPLs in other species. (B) Sequence alignment of GhAGPL1 with its homologs in Arabidopsis thaliana, Citrullus lanatus, Solanum lycopersicum, Vitis vinifera, and Zea mays. The conserved (a) ATP-binding motif, (b) catalytic motif, (c) Glc-1-phosphate motif, and (d) activator site are represented by red boxes, respectively. 
of GhAGPL1 was fused to the C-terminal of GFP, driven by CaMV35S promoter. Both recombined and unrecombined vectors were transferred into onion epidermal cells by particle bombardment. The results (Fig. 2) showed that GFP signals of the control were observed throughout the cell, whereas the signals of GFP-GhAGPL1 were observed in the cytoplasm.

EXPRESSION PROFILE OF GHAGPL1. To better understand GhAGPL1, we analyzed its expression profile in different organs and under abiotic stress conditions using qRT-PCR. The data indicates that GhAGPL1 was expressed constitutively in different organs, and was largely expressed in sink organs (corms and cormels) especially cormels (Fig. 3A). When treated with sucrose, mannitol, or glucose, the transcription level of GhAGPL1 increased dramatically. However, ABA had a negative effect on GhAGPL1 expression, inhibiting its transcription (Fig. 3B).

GHAGPL1 CONTRIBUTES TO STARCH SYNTHESIS IN ARABIDOPSIS. To detect whether GhAGPL1 plays a role in starch synthesis, we complemented the apll arabidopsis mutant (Salk_139228) with the overexpression of GhAGPL1. Three different transgenic complementary lines $(2,6$, and 12$)$

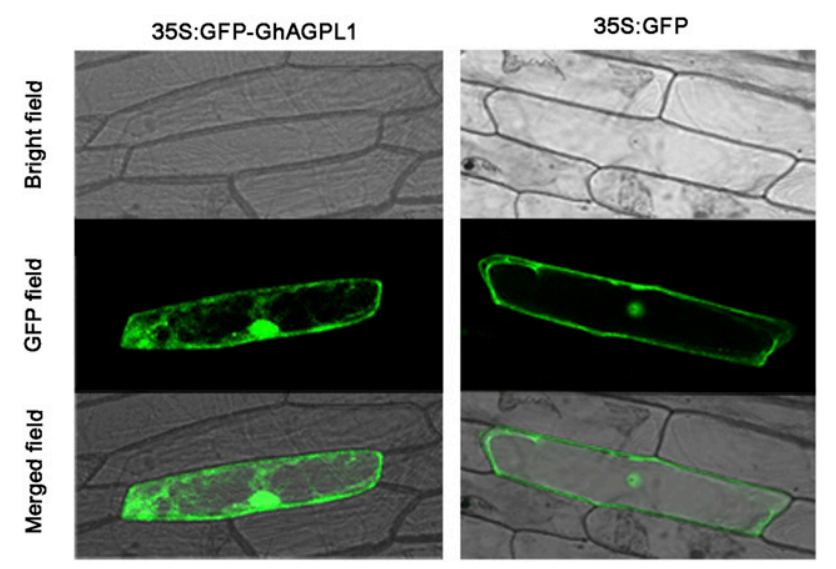

Fig. 2. Subcellular localization of gladiolus GhAGPL1. 35S:GFP-GhAGPL1 (left panels) and 35S:GFP (right panels) were transiently expressed in onion epidermal cells by the particle bombardment.
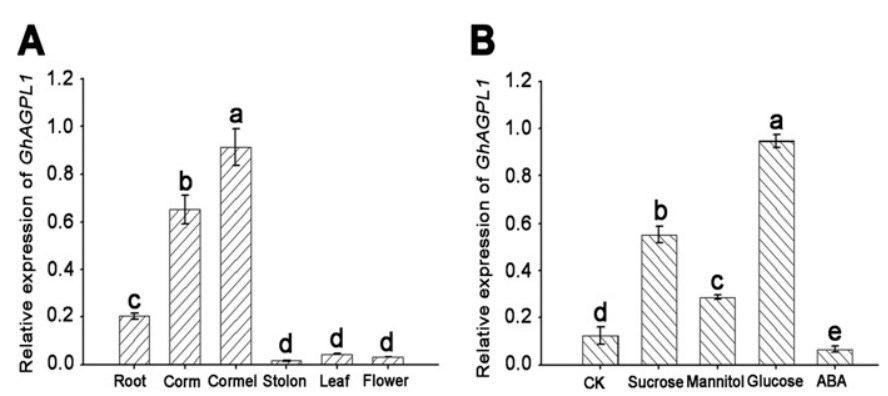

Fig. 3. Quantitative real-time polymerase chain reaction (PCR) analysis of GhAGPL1 expression levels in various gladiolus organs and different treatments. (A) GhAGPL1 expression levels in various gladiolus organs (root, corm, cormel, stolon, leaf, flower). (B) GhAGPL1 expression levels under different abiotic stress. For abiotic stress, cormels were placed in a petri plate in the sugar-free medium containing $200 \mathrm{~mm}$ mannitol, $200 \mathrm{~mm}$ glucose, $200 \mathrm{~mm}$ sucrose, or $100 \mu \mathrm{m}$ abscisic acid (ABA), respectively. Different letters indicate significant differences (Duncan's multiple range test at $P<0.05$ ); identical letters indicate no statistical difference. Actin was used as the internal control to normalize PCR efficiency (Larionov et al., 2005). were chosen for further study (Fig. 4A). As apl1 is a transfer DNA null mutant, it had low starch content resulting from low AGPase activity (Supplemental Fig. 1). However, AGPase activity was dramatically higher in the complementary lines. The level of AGPase activity was correlated with the transcript level of GhAGPL1, although its activity was not as high as wild type [WT (Fig. 4B)].

Next, we investigated the effect of GhAGPL1 on starch synthesis. Starch accumulation in WT and complementary lines followed a similar pattern (Fig. 4C): starch content increased throughout the day, and reached its peak at the end of the day, after which the starch content decreased quickly throughout the night.

Furthermore, we explored the relationship between GhAGPL1 and fresh weight. In the first 3 weeks, the fresh weight of all arabidopsis lines was similar. The growth and development of all arabidopsis lines was slow. After 4 weeks, there was a great difference between rescued lines and apll mutant. The fresh weight of the apll mutant was only $0.99 \mathrm{~g}$, whereas the fresh weight of the rescued lines was similar to WTs (Fig. 4D). These results showed GhAGPL1 could rescue the slower growth rate of arabidopsis apll mutant.

Taken together, these results indicate that GhAGPL1 shows conserved function to AtAGPL1 and its overexpression promotes AGPase activity, starch synthesis, and fresh weight in arabidopsis.

Silencing of GhagPL1 reduces AGPase activity and STARCH SYNTHESIS IN GLADIOLUS. Intensive studies focused on function of $A G P L$ in perennial and herbaceous plants, but there is little information about AGPL function in corm organs. Therefore, we used VIGS to eliminate GhAGPL1 gene expression in
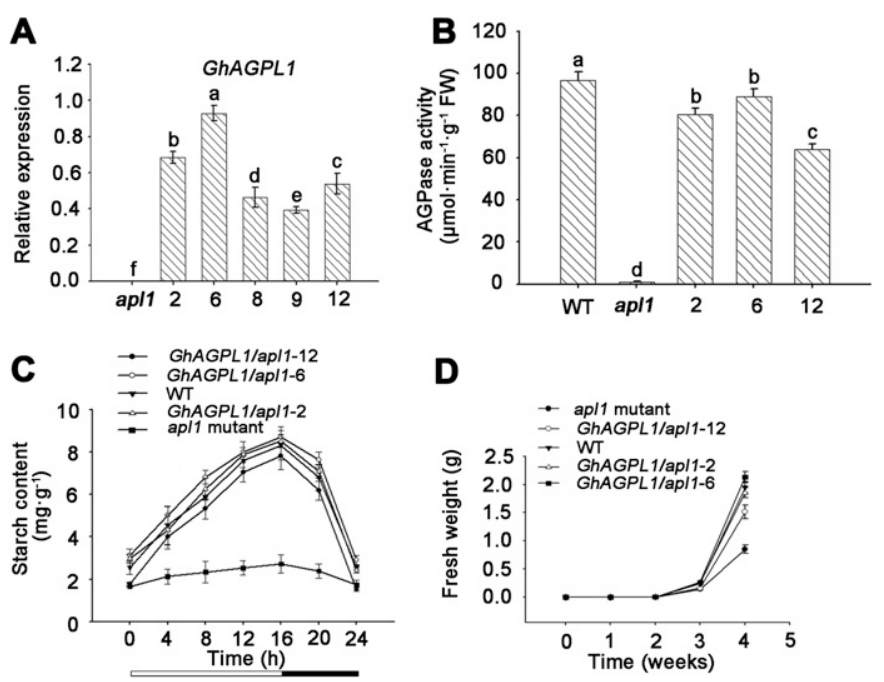

Fig. 4. GhAGPL1 complemented starch synthesis defect in apll arabidopsis mutant. (A) Transcriptional analysis of GhAGPL1 in apll and complementary lines (35S:GhAGPL1/apl1-2, 6, 8, 9, 12), (B) AGPase activity, (C) starch content, and (D) fresh weight in wild type (WT), apll mutant, and complementary lines (35S:GhAGPL1/apl1-2, 6, 12). WT was arabidopsis Columbia (Col) ecotype. (C) Starch content in leaves during a 16/8 h light/ dark photoperiod. The bar at the bottom of the graph represents day (white) and night (black). (D) Fresh weight per plant plotted against time after germination. Leaves were sampled at the end of the light period. The error bars represent \pm SD of data from three biological replicates. Different letters indicate significant differences (Duncan's multiple range test at $P<0.05$ ); identical letters indicate no statistical difference. 
gladiolus. First, we detected the existence of tobacco (Nicotiana tabacum) rattle virus by RT-PCR after agroinfiltration of sprouted cormels. Compared with the control, a larger PCR product was observed in TRV-GhAGPL1-treated groups (Fig. $5 \mathrm{~A}$ ), indicating successful infection of gladiolus.

Then we performed qRT-PCR to select silenced plants. The expression of GhAGPL1 was found to be significantly lower in several silenced plants (Fig. 5B). In addition, we also tracked the expression of GhSus. GhSus encodes sucrose synthase (Sus), an enzyme which plays an important role in conversion of sucrose to UDP-glucose in sink organs, a necessary step for starch synthesis. In GhAGPL1-silenced plants, GhSus expression was $18 \%$ lower compared with control (Fig. 5B), suggesting GhAGPL1 accumulation may play a role in the upstream feedback regulation of GhSus transcription.

To investigate the effect of silencing GhAGPL1 in gladiolus starch synthesis, we measured AGPase activity, starch content, and sucrose content. AGPase activity in GhAGPL1-silenced corm was reduced by around $25 \%$, and it further decreased in
A

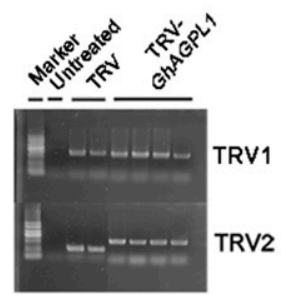

C
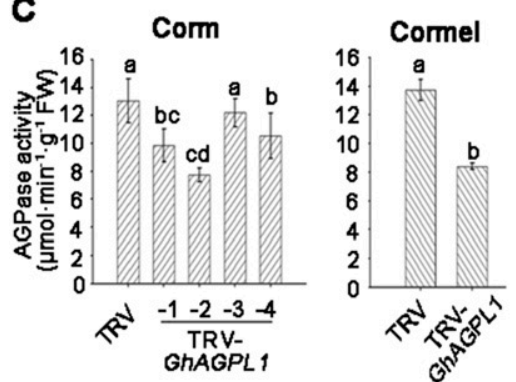

E

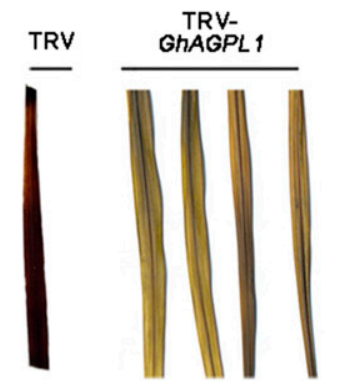

B

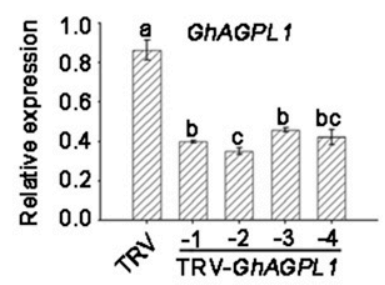

D

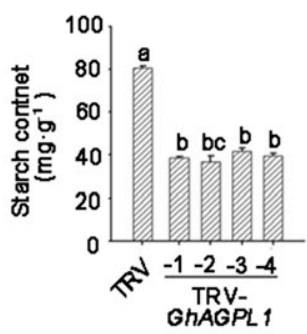

$\mathbf{F}$

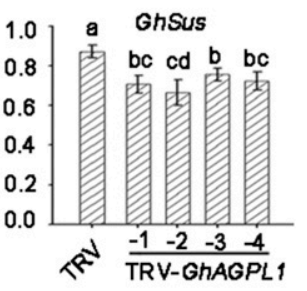

silenced cormels (Fig. 5C). Starch accumulation in silenced corms was around a half, compared with the control (Fig. 5D). Using transmission electron microscopy, we found fewer and smaller starch bodies in silenced corm than the control (Fig. 5F). A similar situation was observed in silenced leaves (Fig. $5 \mathrm{E}$ and $\mathrm{F}$ ). In contrast, sucrose content was $22 \%$ higher in silenced corms. Thus, silencing GhAGPL1 has a negative effect on starch accumulation.

Silencing GhAGPL1 decreases the Quality and QUANTITY OF GLADIOLUS CORMS AND CORMELS. The onset of corm formation is accompanied with starch accumulation. Therefore, we investigated whether inhibition of GhAGPL1 expression would result in any change with respect to corm formation and development.

Plants from the control and silenced lines were harvested at the end of cultivation (when leaves were totally withered). We analyzed their corm weight, corm size, cormel number, and cormel weight. Although there was no visual difference in corm diameter, fresh weight and dry weight were reduced by $16 \%$ and $24 \%$ in silenced corms, respectively (Fig. 6A). For cormels, there was a significant reduction in fresh weight and dry weight (Fig. 6B). In addition, the number of cormels was only $14 \%$ of the control (Fig. $6 \mathrm{~B}$ and C). These results indicate that a reduction in GhAGPL1 is paralleled by a decrease in the fresh weight and dry weight of corm or cormels, and is correlated with a lower number of cormels.

\section{Discussion}

Corm degeneration is a severe problem in the production of gladiolus cut flowers. Degenerated corms produce slim and branched inflorescences, and fewer cormels, which do not meet the standards of commercial sales and propagation (Seng et al., 2016). In addition, degeneration also confers weak disease resistance which leads to great economic loss. Degeneration also occurs in sweetpotato [Ipomoea batatas (Adikini et al., 2015)]. Hence, the quality and quantity of corms and cormels predominantly influence the commercial cut flower industry.

Over decades, $A G P L$ genes were studied intensively in annual plants such as arabidopsis, O. sativa, T. aestivum, and $Z$. mays (Huang et al., 2011; Meyer et al., 2004; Tuncel et al., 2014; Ventriglia et al., 2008). However, since they do not have modified organs, we still do not know much about the function of $A G P L$ in corm. In our study, we silenced GhAGPL1 in gladiolus,

Fig. 5. Downregulation of GhAGPL1 decreases starch biosynthesis and increases sucrose content in gladiolus. (A) Detection of TRV1 and TRV2 in untreated, TRV and TRV-GhAGPL1 plants (TRV-GhAGPL1-1, TRVGhAGPL1-2, TRV-GhAGPL1-3, TRV-GhAGPL1-4). TRV1 is partial TRV, containing its movement protein, whereas TRV2 contains the coat protein. TRV control refers to empty TRV2. TRV-GhAGPL1 plant: GhAGPL1silenced plant. (B) Transcription analysis of GhAGPL1 and GhSus in TRV and four TRV-GhAGPL1 lines. (C) AGPase activity in TRV and silenced corm and cormels. (D) Starch and sucrose content in TRV and four TRVGhAGPL1 lines. (E) The phenotype of gadiolus leaves in TRV and TRV-GhAGPL1 lines via Lugol's iodine stain. (F) Transmission electron microscopy of leaf and corm section from TRV and four TRV-GhAGPL1 lines. Different letters indicate significant differences (Duncan's multiple range test at $P<0.05$ ); identical letters indicate no statistical difference. 

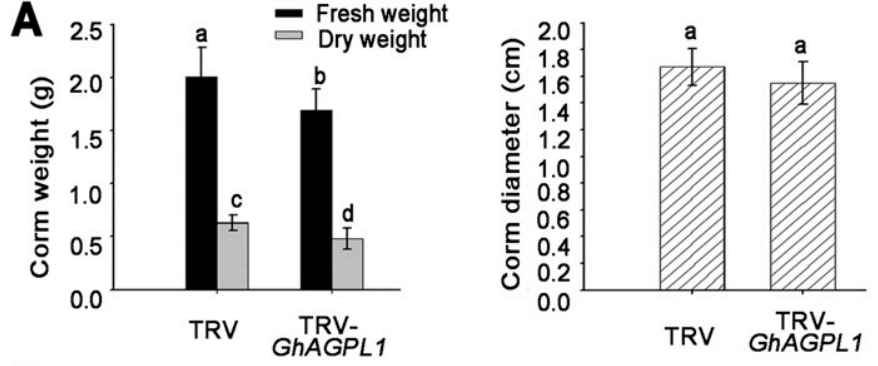

B
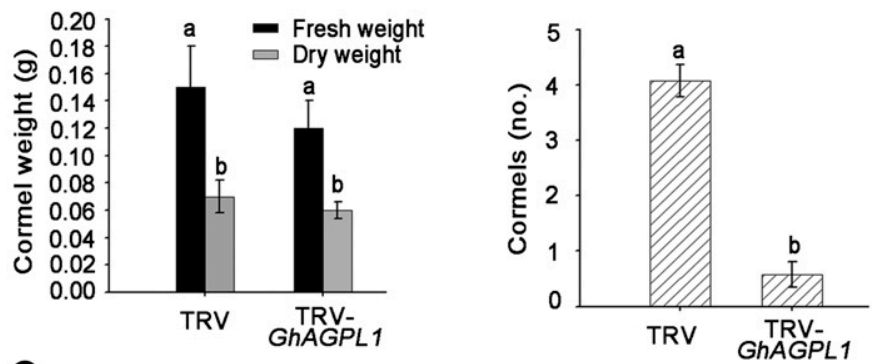

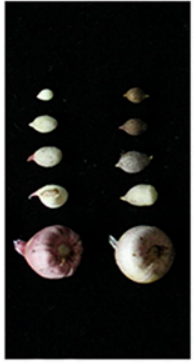

TRV

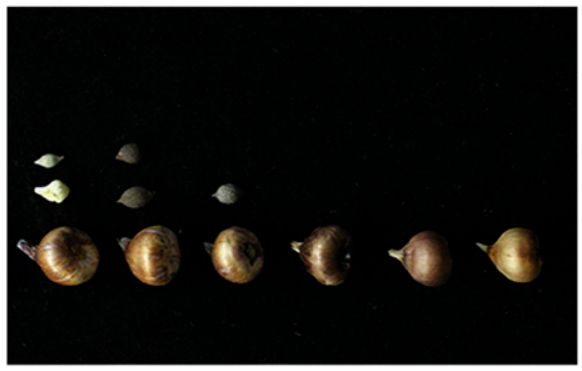

TRV-GhAGPL1

Fig. 6. Silencing GhAGPL1 decreases the quality and quantity of gladiolus corms and cormels. (A) Fresh weight, dry weight of corm, and corm diameter in TRV and four TRV-GhAGPL1 lines. (B) Fresh weight, dry weight of cormel, and cormel number in TRV and four TRV-GhAGPL1 lines. (C) Silencing GhAGPL1 in gladiolus leads to fewer number of cormels. Different letters indicate significant differences (Duncan's multiple range test at $P<0.05$ ); identical letters indicate no statistical difference.

and found a reduction in corm weight and cormel number (Fig. 6). Previously, studies have shown that an increase in the amount of sucrose within an appropriate range, promotes gladiolus corm formation, diameter, and fresh weight (Dantu and Bhojwani, 1995). The weight and the number of cormels were closely correlated with mother corm size in brodiaea [Brodiaea laxa (Han et al., 1991)]. Our results are in agreement with their research. We provide molecular evidence that GhAGPL1 plays a vital role in corm development. In addition, we find a distinct role of $A G P L$ in gladiolus and potato (Solanum tuberosum). In potato, silencing AGPase B reduced tuber size but resulted in an increase in both total tuber number and the number of tubers formed per stolon (Müller-Röber et al., 1992). We hypothesize that this difference may result from different mechanisms of tuberization and cormel initiation. However, they do share similarities, such as weak sink ability in silenced tubers or corms.

Downregulating GhAGPL1 via VIGS leads to low expression of GhSus, but resulted in higher sucrose accumulation in gladiolus (Fig. 5). Silencing GhAGPL1 reduced AGPase activity, resulting in low level of sucrose availability and reduction of starch content. Furthermore, silencing GhAGPL1 reduced the transcription level of GhSus and promoted sucrose accumulation (Fig. 5). AGPase B-silenced lines of potato also have lower amount of starch, higher amount of glucose and sucrose, and lower expression of Sus (Müller-Röber et al., 1992).

In this study, GhAGPL1 was mostly expressed in sink organs, while being expressed lower in source organs [leaf (Fig. 3A)]. This expression profile promotes sucrose-to-starch conversion in sink organs (Yang et al., 2004). GhAGPL1 expression was suppressed by ABA (Fig. 3B), which is consistent with other research that shows $\mathrm{ABA}$ alone can reduce starch accumulation in O. sativa (Akihiro et al., 2005). Furthermore, sucrose and glucose induced GhAGPL1 expression (Fig. 3B), which is similar to its homologs in arabidopsis (Sokolov et al., 1998).

In summary, we have isolated and characterized a large subunit of AGPase in gladiolus. GhAGPL1 was highly expressed in sink organs (cormel and corm), and was induced by sucrose, glucose, and mannitol, while being repressed by ABA. Silencing GhAGPL1 in gladiolus resulted in reduction of corm yield and number of cormels.

\section{Literature Cited}

Adikini, S., S.B. Mukasa, R.O.M. Mwanga, and R.W. Gibson. 2015. Sweet potato cultivar degeneration rate under high and low sweet potato virus disease pressure zones in Uganda. Can. J. Plant Pathol. 37:136-147.

Akihiro, T., K. Mizuno, and T. Fujimura. 2005. Gene expression of ADP-glucose pyrophosphorylase and starch contents in rice cultured cells are cooperatively regulated by sucrose and ABA. Plant Cell Physiol. 46:937-946.

Butt, S.J. 2005. Effect of N, P, K on some flower quality and corm yield characteristics of gladiolus. J. Tekirdag Agr. Faculty 2:212-214.

Caspar, T., S.C. Huber, and C. Somerville. 1985. Alterations in growth, photosynthesis, and respiration in a starchless mutant of Arabidopsis thaliana L. deficient in chloroplast phosphoglucomutase activity. Plant Physiol. 79:11-17.

Chen, J. and M.F. Yi. 2004. Effects of different planting time on growth and development of gladiolus stolon and cormel. Acta Hort. 31:767-772.

Clough, S.J. and A.F. Bent. 1998. Floral dip: A simplified method for agrobacterium-mediated transformation of Arabidopsis thaliana. Plant J. 16:735-743.

Crevillén, P., M.A. Ballicora, Á. Mérida, J. Preiss, and J.M. Romero. 2003. The different large subunit isoforms of Arabidopsis thaliana ADP-glucose pyrophosphorylase confer distinct kinetic and regulatory properties to the heterotetrameric enzyme. J. Biol. Chem. 278:28508-28515.

Crevillén, P., T. Ventriglia, F. Pinto, A. Orea, Á. Mérida, and J.M. Romero. 2005. Differential pattern of expression and sugar regulation of Arabidopsis thaliana ADP-glucose pyrophosphorylaseencoding genes. J. Biol. Chem. 280:8143-8149.

Cross, J.M., M. Clancy, J.R. Shaw, T.W. Greene, R.R. Schmidt, T.W. Okita, and L.C. Hannah. 2004. Both subunits of ADP-glucose pyrophosphorylase are regulatory. Plant Physiol. 135:137-144.

Dantu, P.K. and S.S. Bhojwani. 1995. In vitro corm formation and field evaluation of corm-derived plants of gladiolus. Sci. Hort. 61:115129.

Gonzalez-Melendi, P., M. Uyttewaal, C.N. Morcillo, J.R.H. Mora, S. Fajardo, F. Budar, and M.M. Lucas. 2008. A light and electron microscopy analysis of the events leading to male sterility in OguINRA CMS of rapeseed (Brassica napus). J. Expt. Bot. 59:827-838. Hall, T.A. 1999. BioEdit: A user-friendly biological sequence alignment editor and analysis program for Windows 95/98/NT. Nucl. Acids Symp. Ser. 41:95-98. 
Han, S.S., A.H. Halevy, R.M. Sachs, and M.S. Reid. 1991. Flowering and corm yield of brodiaea in response to temperature, photoperiod, corm size, and planting depth. J. Amer. Soc. Hort. Sci. 116:19-22.

Hendriks, J.H.M., A. Kolbe, Y. Gibon, M. Stitt, and P. Geigenberger. 2003. ADP-glucose pyrophosphorylase is activated by posttranslational redox-modification in response to light and to sugars in leaves of arabidopsis and other plant species. Plant Physiol. 133:838-849.

Huang, B.Q., J. Chen, J.J. Zhang, H.M. Liu, M.L. Tian, Y. Gu, Y.F. Hu, Y.P. Li, Y.H. Liu, and Y.B. Huang. 2011. Characterization of ADPglucose pyrophosphorylase encoding genes in source and sink organs of maize. Plant Mol. Biol. Rpt. 29:563-572.

Kang, G., G. Liu, X. Peng, L. Wei, C. Wang, Y. Zhu, Y. Ma, Y. Jiang, and T. Guo. 2013. Increasing the starch content and grain weight of common wheat by overexpression of the cytosolic AGPase large subunit gene. Plant Physiol. Biochem. 73:93-98.

Larionov, A., A. Krause, and W. Miller. 2005. A standard curve based method for relative real time PCR data processing. BMC Bioinformatics 6:62.

Livak, K.J. and T.D. Schmittgen. 2001. Analysis of relative gene expression data using real-time quantitative PCR and the $2^{-\triangle \triangle C} C_{T}$ method. Methods 25:402-408.

Meyer, F.D., E.D. Smidansky, B. Beecher, T.W. Greene, and M.J. Giroux. 2004. The maize Sh2r6hs ADP-glucose pyrophosphorylase (AGP) large subunit confers enhanced AGP properties in transgenic wheat (Triticum aestivum). Plant Sci. 167:899-911.

Müller-Röber, B., U. Sonnewald, and L. Willmitzer. 1992. Inhibition of the ADP-glucose pyrophosphorylase in transgenic potatoes leads to sugar-storing tubers and influences tuber formation and expression of tuber storage protein genes. EMBO J. 11:1229-1238.

Roldan, I., F. Wattebled, M.M. Lucas, D. Delvalle, V. Planchot, S. Jimenez, R. Perez, S. Ball, C. D’Hulst, and A. Merida. 2007. The phenotype of soluble starch synthase IV defective mutants of Arabidopsis thaliana suggests a novel function of elongation enzymes in the control of starch granule formation. Plant J. 49:492-504.

Saripalli, G. and P.K. Gupta. 2015. AGPase: Its role in crop productivity with emphasis on heat tolerance in cereals. Theor. Appl. Genet. 128:1893-1916.

Seng, S.S., J. Wu, J.J. Sui, C.Y. Wu, X.H. Zhong, C. Liu, C. Liu, B.H. Gong, F.Q. Zhang, J.N. He, and M.F. Yi. 2016. ADP-glucose pyrophosphorylase gene plays a key role in the quality of corm and yield of cormels in gladiolus. Biochem. Biophys. Res. Commun. 474:206-212. Sharma, J.R. and R.B. Gupta. 2003. Effect of corm size and spacing on growth, flowering and corm production in gladiolus. J. Ornamental Hort. 6:352-356.
Sokolov, L.N., A. Dejardin, and L.A. Kleczkowski. 1998. Sugars and light/dark exposure trigger differential regulation of ADP-glucose pyrophosphorylase genes in Arabidopsis thaliana (thale cress). Biochem. J. 336:681-687.

Tamura, K., D. Peterson, N. Peterson, G. Stecher, M. Nei, and S. Kumar. 2011. MEGA5: Molecular evolutionary genetics analysis using maximum likelihood, evolutionary distance, and maximum parsimony methods. Mol. Biol. Evol. 28:2731-2739.

Thompson, J.D., T.J. Gibson, F. Plewniak, F. Jeanmougin, and D.G. Higgins. 1997. The ClustalX windows interface: Flexible strategies for multiple sequence alignment aided by quality analysis tools. Nucleic Acids Res. 24:4876-4882.

Tuncel, A., B. Cakir, S.K. Hwang, and T.W. Okita. 2014a. The role of the large subunit in redox regulation of the rice endosperm ADPglucose pyrophosphorylase. FEBS J. 281:4951-4963.

Tuncel, A., J. Kawaguchi, Y. Ihara, H. Matsusaka, A. Nishi, T. Nakamura, S. Kuhara, H. Hirakawa, Y. Nakamura, B. Cakir, A. Nagamine, T.W. Okita, S.K. Hwang, and H. Satoh. 2014b. The rice endosperm ADP-glucose pyrophosphorylase large subunit is essential for optimal catalysis and allosteric regulation of the heterotetrameric enzyme. Plant Cell Physiol. 55:1169-1183.

Ventriglia, T., M.L. Kuhn, M.T. Ruiz, M.R. Pedro, F. Valverde, M.A. Ballicora, J. Preiss, and J.M. Romero. 2008. Two arabidopsis ADPglucose pyrophosphorylase large subunits (APL1 and APL2) are catalytic. Plant Physiol. 148:65-76.

Wang, G.L. and H.Y. Fang. 2002. Gene engineering in plant. 2nd ed. Press Sci., Beijing, China.

Wu, J., S.S. Seng, J.J. Sui, V. Eliana, X. Luo, B.H. Gong, C. Liu, C.Y. Wu, C. Liu, F.Q. Zhang, J.N. He, and M.F. Yi. 2015. Gladiolus hybridus abscisic acid insensitive $5(G h A B I 5)$ is an important transcription factor in $\mathrm{ABA}$ signaling that can enhance gladiolus corm dormancy and arabidopsis seed dormancy. Front. Plant Sci. 6:749-753.

Yang, J.C., J.H. Zhang, Z.Q. Wang, G.W. Xu, and Q.S. Zhu. 2004. Activities of key enzymes in sucrose-to-starch conversion in wheat grains subjected to water deficit during grain filling. Plant Physiol. 135:1621-1629.

Yu, S.M., S.F. Lo, and T.H. Ho. 2015. Source-sink communication: Regulated by hormone, nutrient, and stress cross-signaling. Trends Plant Sci. 20:844-857.

Zhong, X.H., X. Yuan, Z. Wu, M.A. Khan, J. Chen, X. Li, B.H. Gong, Y. Zhao, J. Wu, C. Wu, and M.F. Yi. 2014. Virus-induced gene silencing for comparative functional studies in Gladiolus hybridus. Plant Cell Rpt. 33:301-312. 

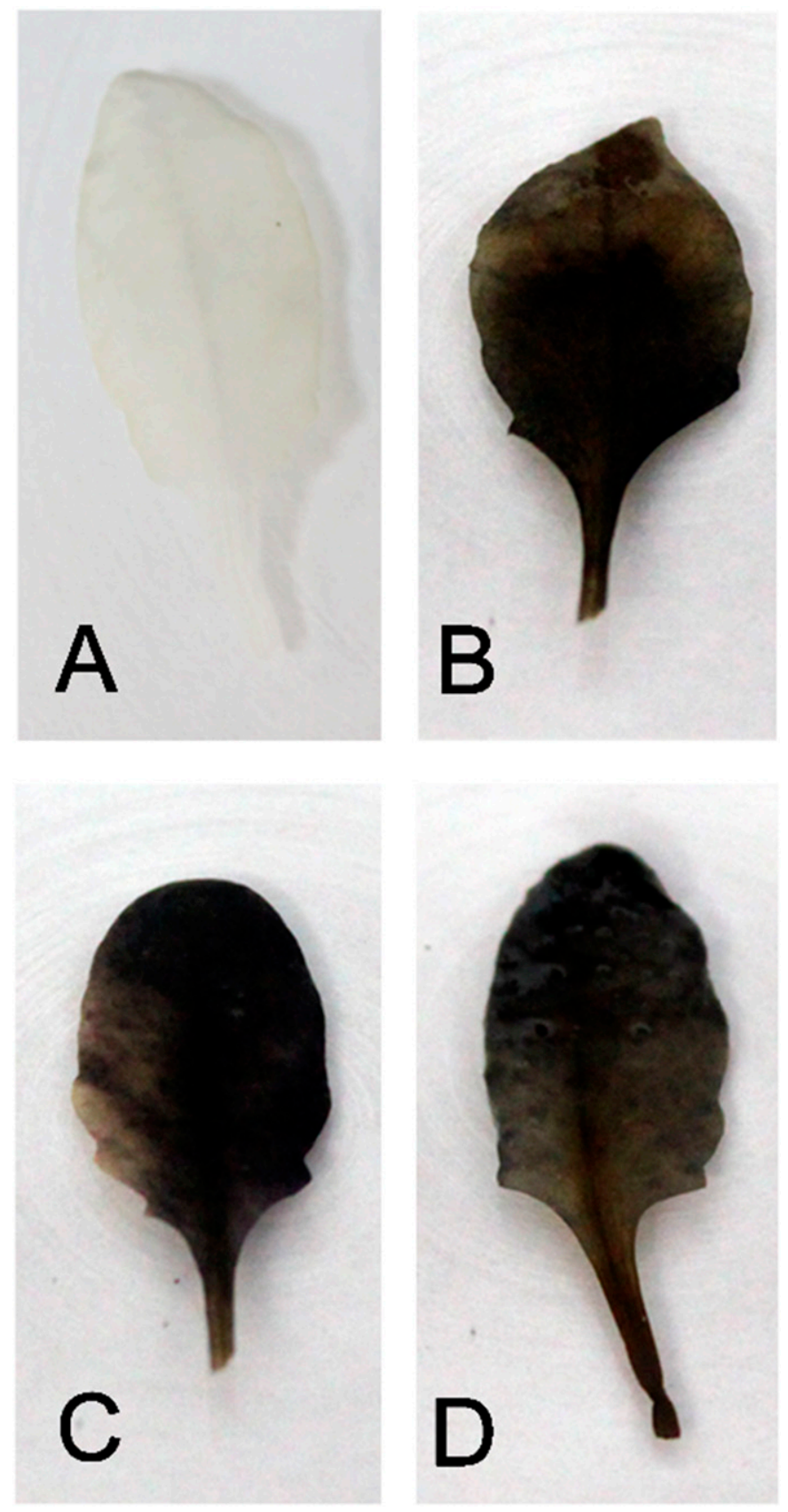

Supplemental Fig. 1. Iodine staining of arabidopsis leaves of apll mutants and complementary lines. Rosette leaves from plants grown for 3 weeks were stained with lugol. (A) ap1 mutant line: Salk-139228; (B) complementary line: 35S:AGPL1/apl1-2; (C) complementary line: 35S:AGPL1/apl1-6; and (D) complementary line: 35S:AGPL1/apl1-12. 
Supplemental Table 1. Primers used in this study.

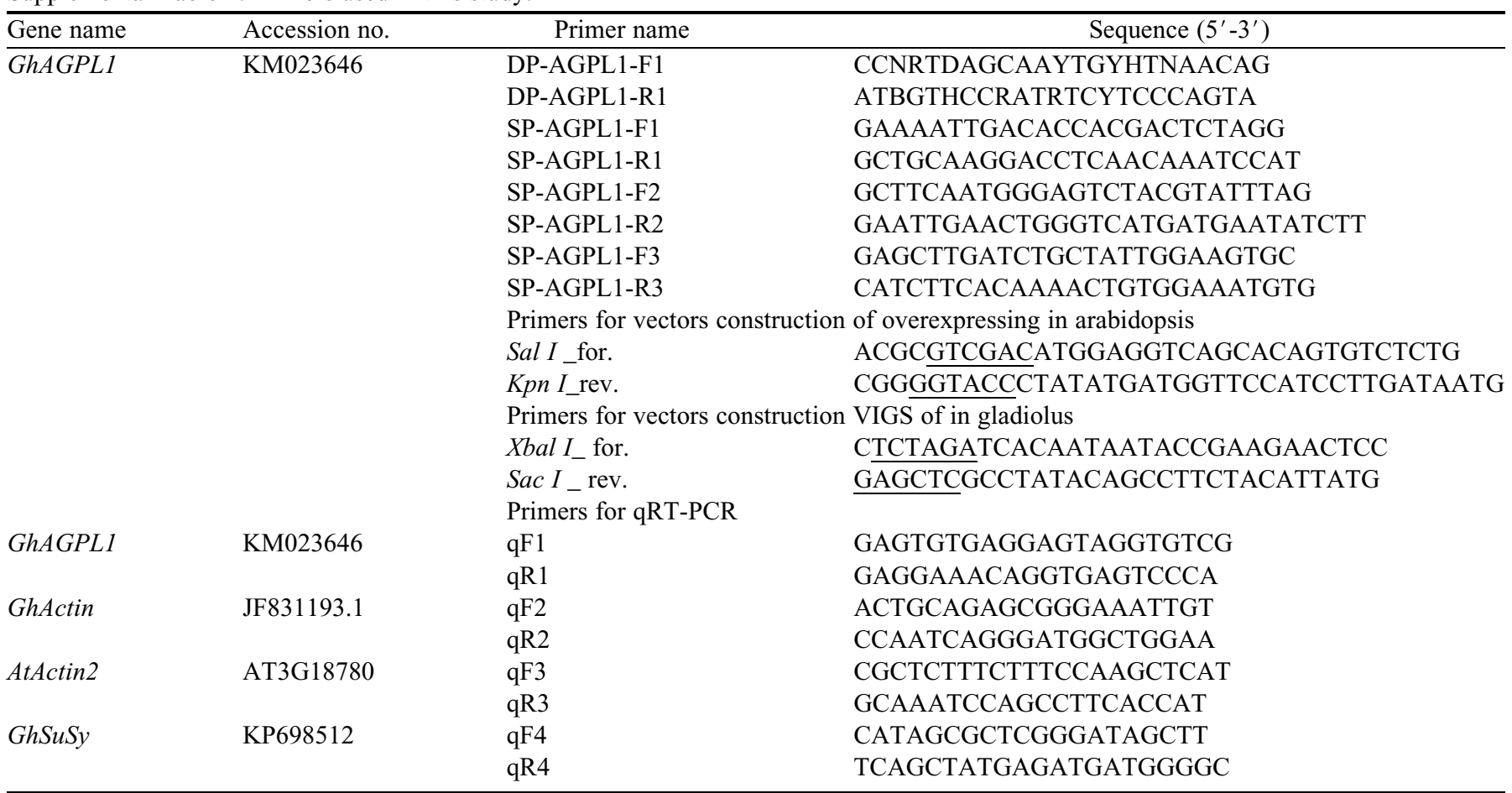

VIGS = virus-induced gene silencing; qRT-PCR = quantitative real-time polymerase chain reaction . 
Supplemental Table 2. GhAGPL1 homologs used in the sequence analysis from NCBI database.

\begin{tabular}{|c|c|c|}
\hline Species & Name & Accession no. \\
\hline Actinidia deliciosa & Actinidia deliciosa 4 & AGS94412.1 \\
\hline Arabidopsis thaliana & Arabidopsis thaliana 1 & AAM14190 \\
\hline A. thaliana & Arabidopsis thaliana 2 & BAA76362 \\
\hline A. thaliana & Arabidopsis thaliana 3 & AAM20291 \\
\hline A. thaliana & Arabidopsis thaliana 4 & CAA77173 \\
\hline Beta vulgaris & Beta vulgaris & CAA55516 \\
\hline B. vulgaris & Beta vulgaris 1 & AGB85111.1 \\
\hline Brassica rapa & Brassica rapa & AAK27685 \\
\hline Cicer arietinum & Cicer arietinum 1 & AAK27719 \\
\hline C. arietinum & Cicer arietinum 2 & AAK 27718 \\
\hline Citrullus lanatus & Citrullus lanatus 1 & AAB91468 \\
\hline C. lanatus & Citrullus lanatus 2 & AAB91467 \\
\hline Citrus unshiu & Citrus unshiu & AAD56042 \\
\hline Cucumis melo & Cucumis melo 1 & AAB91463 \\
\hline C. melo & Cucumis melo 2 & AAB91464 \\
\hline Fragaria ananassa & Fragaria ananassa 1 & AAS00542 \\
\hline Gladiolus hybridus & GhAGPL1 & AIO11223.1 \\
\hline Hordeum vulgare & Hordeum vulgare 1 & CAA47626 \\
\hline H. vulgare & Hordeum vulgare 2 & AAC49729 \\
\hline Ipomoea batatas & Ipomoea batatas 2 & AGB85110.1 \\
\hline I. batatas & Ipomoea batatas 3 & AGB85111.1 \\
\hline I. batatas & Ipomoea batatas 4 & AGB85112.1 \\
\hline Oncidium goldiana & Oncidium goldiana & AAM95945 \\
\hline Oryza sativa & Oryza sativa 1 & BAA23490 \\
\hline O. sativa & Oryza sativa 2 & AAB38781 \\
\hline O. sativa & Oryza sativa 3 & NP_911710 \\
\hline O. sativa & Oryza sativa 4 & AAT78793 \\
\hline Ostreococcus tauri & Ostreococcus tauri & AAS88891 \\
\hline Perilla frutescens & Perilla frutescens & AAF66436 \\
\hline Phaseolus vulgaris & Phaseolus vulgaris 1 & BAC66692 \\
\hline Pisum sativum & Pisum sativum & CAA65541 \\
\hline Populus trichocarpa & Populus trichocarpa 2 & XP002300758.1 \\
\hline Solanum habrochaites & Solanum habrochaites & AAD56405 \\
\hline Solanum lycopersicum & Solanum lycopersicum 1 & AAC49942 \\
\hline S. lycopersicum & Solanum lycopersicum 2 & AAC49941 \\
\hline S. lycopersicum & Solanum lycopersicum 3 & AAC49943 \\
\hline Solanum tuberosum & Solanum tuberosum 1 & CAA43490 \\
\hline S. tuberosum & Solanum tuberosum 2 & CAA52917 \\
\hline S. tuberosum & Solanum tuberosum 3 & CAA53741 \\
\hline Sorghum bicolor & Sorghum bicolor & AAB94012 \\
\hline Triticum aestivum & Triticum aestivum & CAA79980 \\
\hline Vitis vinifera & Vitis vinifera 1 & XP_010664711.1 \\
\hline$V$. vinifera & Vitis vinifera 2 & XP_010652349.1 \\
\hline Zea mays & Zea mays 1 & JX462603.1 \\
\hline Z. mays & Zea mays 2 & CAA86227 \\
\hline Z. mays & Zea mays 3 & EU971227.1 \\
\hline
\end{tabular}

